\section{Acute kidney injury}

Simon Lines, Specialist Registrar; Andrew Lewington, Consultant Renal Physician/Honorary Senior Lecturer

Renal Department, St James's University Hospital, Leeds

The term acute kidney injury (AKI) has now replaced the term acute renal failure (ARF) and represents the entire spectrum of the latter. It is best characterised by a rapid decline in kidney function over hours to weeks with the retention of nitrogenous waste products and creatinine. The adoption of the new term for $\mathrm{ARF}$ and proposals for a universal definition and classification system for AKI is the result of a collaborative effort by the Acute Dialysis Quality Initiative (ADQI) and the Acute Kidney Injury Network (AKIN). ${ }^{1,2}$ Concurrently there has been growing interest in AKI, particularly with respect to the increased mortality rate associated with minor rises in serum creatinine (SCr). ${ }^{3,4}$ This article will initially consider the entire spectrum of AKI for clinical purposes, but then concentrate on one of the most commonly encountered form, acute tubular necrosis (ATN).

\section{Definitions}

Progress in understanding AKI has been hampered by the lack of a universal definition. Over 35 different definitions have been used to define ARF in the literature. Major advances towards developing a universally accepted definition were taken in 2002 when the ADQI proposed the Risk, Injury and Failure, Loss of function and End-stage kidney disease (RIFLE) staging system (Table 1). The classification thus includes three levels of increasing kidney dysfunction and two outcomes. Categorisation into a particular stage depends upon rises in SCr from baseline or reductions in glomerular filtration rate (GFR) within a one-week time interval or reductions in urine output.

Further studies subsequently demonstrated that relatively small increments in $\mathrm{SCr}$ are associated with a significant increase in patient mortality. This prompted proposals by AKIN in $2005,{ }^{2}$ to modify the RIFLE staging system to produce an interim staging system allowing additional data to be gathered and research initiatives proposed (Table 1). Further refinement of the definition based upon the results of future studies is anticipated.

\section{Epidemiology}

The incidence of AKI depends upon the definition used and the cohort studied. Accurate assessment is difficult due to the lack of a standardised registry. Based on previous studies, AKI has been estimated to occur in $3-7 \%$ of hospitalised patients. ${ }^{5}$ More recently, a study using the RIFLE staging system identified that $18 \%$ of patients admitted to a large urban medical centre had evidence of AKI. ${ }^{6}$ The incidence of AKI on the intensive care unit (ICU) has been characterised more clearly due to established data collection systems and estimated at $25-30 \% .^{5}$ The
RIFLE staging system has demonstrated a wide ranging incidence of AKI dependent upon the population case mix. ${ }^{5}$

\section{Aetiology}

The aetiology of AKI is best considered divided into three main categories (Table 2):

- pre-kidney

- intrinsic kidney, and

- post-kidney.

This division allows the clinician to consider the cause of the injury systematically for the purposes of diagnosis and management. The frequency of aetiology type will depend upon the cohort studied. Outside the ICU the aetiology of patients with AKI is generally recognised as pre-kidney $55-60 \%$, intrinsic $35-40 \%$ and post-kidney $<5 \% .{ }^{7}$ In contrast, on the ICU most critically ill patients with multi-organ failure will have potentially reversible ischaemic intrinsic AKI secondary to kidney hypoperfusion.

Table 1. Definition and classification schemes for acute kidney injury.*

\title{
Criteria
}

\begin{tabular}{|c|c|c|}
\hline & Serum creatinine & Urine output \\
\hline \multicolumn{3}{|l|}{ RIFLE } \\
\hline Risk & $\uparrow \mathrm{SCr} \geq 1.5 \times$ from baseline or $\downarrow$ GFR $\geq 25 \%$ & $<0.5 \mathrm{ml} / \mathrm{kg} / \mathrm{h} \geq 6 \mathrm{~h}$ \\
\hline Injury & $\uparrow \mathrm{SCr} \geq 2.0 \times$ from baseline or $\downarrow$ GFR $\geq 50 \%$ & $<0.5 \mathrm{ml} / \mathrm{kg} / \mathrm{h} \geq 12 \mathrm{~h}$ \\
\hline \multirow[t]{2}{*}{ Failure } & $\begin{array}{l}\uparrow S C r \geq 3.0 \times \text { from baseline or } \downarrow \text { GFR } \geq 75 \% \\
\text { or an acute } \uparrow S C r \geq 44 \mu \mathrm{mol} / / \text { from baseline }\end{array}$ & $<0.3 \mathrm{ml} / \mathrm{kg} / \mathrm{h} \geq 24 \mathrm{~h}$ or anuria $\geq 12 \mathrm{~h}$ \\
\hline & $\mathrm{SCr} \geq 354 \mu \mathrm{mol} / \mathrm{l}$ & \\
\hline Loss & Complete loss of kidney function $>4$ weeks & \\
\hline ESKD & ESKD $>3$ months & \\
\hline \multicolumn{3}{|c|}{$\operatorname{AKIN}^{\star *}$} \\
\hline \multicolumn{3}{|c|}{ Stage $1 \uparrow S C r \geq 1.5 \times$ or $\uparrow S C r 25 \mu \mathrm{mol} / /$ from baseline $<0.5 \mathrm{ml} / \mathrm{kg} / \mathrm{h} \geq 6 \mathrm{~h}$} \\
\hline Stage 2 & $\uparrow \mathrm{SCr} \geq 2.0 \times$ from baseline & $<0.5 \mathrm{ml} / \mathrm{kg} / \mathrm{h} \geq 12 \mathrm{~h}$ \\
\hline Stage 3 & $\begin{array}{l}\uparrow S C r \geq 3.0 \times \text { from baseline or an acute } \uparrow S C r \\
\geq 44 \mu \mathrm{mol} / / \text { from baseline } \mathrm{SCr} \geq 354 \mu \mathrm{mol} / \mathrm{l} \text { o } \\
\text { initiated on RRT (irrespective of stage at time } \\
\text { of initiation) }\end{array}$ & $<0.3 \mathrm{ml} / \mathrm{kg} / \mathrm{h} \geq 24 \mathrm{~h}$ or anuria $\geq 12 \mathrm{~h}$ \\
\hline
\end{tabular}

*Only one criteria (serum creatinine (SCr), glomerular filtration rate (GFR) or urine output) has to be fulfilled to qualify for a specific category. Baseline $\mathrm{SCr}$ is considered to be within one week for RIFLE and within 48 hours for AKIN.

${ }^{* *}$ AKIN criteria only applies after adequate fluid resuscitation has been performed and obstruction excluded.

AKIN = Acute Kidney Injury Network; ESKD = end-stage kidney disease; RIFLE = Risk, Injury and Failure, Loss of function and End-stage kidney disease staging system; RRT = renal replacement therapy. 
Pre-kidney and ischaemic intrinsic AKI may be considered as two extremes of a spectrum of injury that is the result of hypoperfusion of the kidneys. In prekidney AKI there is a reduction in GFR without overt tubule cell injury. Restoration of adequate kidney perfusion will result in rapid recovery of GFR. However, if the hypoperfusion is sustained, ischaemic tubule cell injury occurs resulting in ATN from which recovery will be delayed.

\section{Pathophysiology}

The pathophysiology of AKI will depend upon the underlying aetiology. This article considers only ATN secondary to kidney hypoperfusion. ATN is strictly a histopathological diagnosis, often used interchangeably with ischaemic AKI. Significant kidney hypoperfusion results in the preferential shunting of blood away from the already relatively ischaemic environment of the outer medulla. ${ }^{8}$ Therefore most ischaemic injury is sustained by the proximal tubules and medullary thick ascending limbs of the distal tubules which lie in the outer medulla.

Paradoxically, kidney biopsy studies have failed to demonstrate frank tubule cell necrosis despite marked kidney dysfunction. A number of mechanisms have been proposed to explain the marked reduction in GFR. These include:

- a decrease in the filtration pressure in the glomerulus secondary to afferent arteriolar vasoconstriction and proximal tubular obstruction, and

- back leak of glomerular filtrate across denuded basement membrane and disrupted tubule cell tight junctions.

Recovery of kidney function is possible, involving tubule cell proliferation and differentiation and may take up to six weeks to complete. ${ }^{9}$

Research into the pathophysiology of ischaemic ATN has allowed the discovery of novel biomarkers of kidney injury. ${ }^{10}$ Investigators have identified a number of genes and their gene products that are upregulated in the kidney following ischaemic injury and then secreted into the blood and urine. Such biomarkers may ultimately offer clinicians the opportunity to detect AKI at an earlier stage and enable appropriate therapeutic intervention. Neutrophil gelatinase-associated lipocalin is highly upregulated following ischaemic injury in the human and rapidly excreted into the urine and blood. ${ }^{11}$ The results of a large randomised controlled trial (RCT) are awaited to assess its clinical applicability.

\section{Clinical presentation}

Early differentiation of AKI from chronic kidney disease (CKD) must be performed. The clinician must consider the cause of AKI (Table 2). If intrinsic AKI is diagnosed clinically it must be determined whether this is ischaemic intrinsic AKI or a more esoteric diagnosis. Clinical suspicion of a more esoteric diagnosis (eg vasculitis, intestinal nephritis) should be aroused in patients without a clearly defined insult or who have systemic symptoms. Establishing the diagnosis depends on taking a thorough history (Table 3(a)) and performing a comprehensive clinical examination (Table 3(b)).

\section{Investigations}

Baseline investigations include those listed in Table 4. More specialised investigations

Table 2. Major causes of acute kidney injury (AKI).

\begin{tabular}{|c|c|}
\hline \multicolumn{2}{|l|}{ Pre-kidney } \\
\hline \multirow[t]{4}{*}{ Hypovolaemia } & Haemorrhage \\
\hline & Gastrointestinal losses \\
\hline & Burns \\
\hline & Renal losses \\
\hline \multirow[t]{4}{*}{ Decreased cardiac output } & Cardiac failure \\
\hline & Sepsis \\
\hline & Liver failure \\
\hline & Drugs \\
\hline \multirow[t]{2}{*}{ Pharmacological agents } & ACE inhibitors \\
\hline & NSAIDs \\
\hline \multicolumn{2}{|l|}{ Intrinsic kidney } \\
\hline \multirow[t]{5}{*}{ Large vessel disease } & Thrombosis \\
\hline & Atheroembolism \\
\hline & Thromboembolism \\
\hline & Dissection \\
\hline & Vasculitis \\
\hline Glomerular & Glomerulonephritis \\
\hline \multirow[t]{4}{*}{ Tubular } & Ischaemia (ATN) \\
\hline & Rhabdomyolysis \\
\hline & Myeloma \\
\hline & $\begin{array}{l}\text { Toxins (antibiotics, contrast, } \\
\text { chemotherapeutic agents) }\end{array}$ \\
\hline \multirow[t]{2}{*}{ Interstitial } & Interstitial nephritis \\
\hline & Infiltration (lymphoma) \\
\hline \multicolumn{2}{|l|}{ Post-kidney } \\
\hline \multirow[t]{4}{*}{ Obstruction } & Kidney stones \\
\hline & Retroperitoneal fibrosis \\
\hline & Carcinoma (prostate, cervical) \\
\hline & Catheter \\
\hline Increased abdominal pressure & Intra-abdominal compartment syndrome \\
\hline
\end{tabular}


(Table 5) may be requested if indicated by the clinical presentation. Urine chemistry may help to differentiate prekidney AKI and ATN are shown in Table 6.

\section{Management}

The best form of management of AKI is prevention. This involves careful clinical evaluation of patients and risk stratification (Tables 3(a) and 3(b)). Patients identified to be at risk should have their cardiovascular status and intravascular volume optimised and nephrotoxic drugs avoided. Optimisation of fluid status remains a medical challenge and requires meticulous consideration of the patient's fluid status and requirements. A thorough review is given in the recent British Consensus Guidelines For Intravenous Fluid Therapy In Adult Surgical Patients (GIFTASUP). ${ }^{12}$

High-risk patients with an estimated GFR below $60 \mathrm{ml} / \mathrm{min} / 1.73 \mathrm{~m}^{2}$ undergoing contrast studies should receive volume expansion with $0.9 \%$ sodium chloride at $1 \mathrm{ml} / \mathrm{kg} / \mathrm{hr}$ for 12 hours before the procedure and for several hours afterwards. ${ }^{13}$ There is no compelling evidence to support the use of sodium bicarbonate or $\mathrm{N}$-acetylcysteine to prevent contrast-induced nephropathy, ${ }^{14}$ and similarly none to justify the use of dopamine, loop diuretics or mannitol for the prevention or treatment of AKI.

\section{General supportive therapy}

All patients who develop AKI will require general supportive therapy involving stabilisation of the patient's haemodynamic status and prompt treatment of any underlying disease. AKI is accompanied by a number of complications that may require specific therapy (Table 7). The severity of the complications will tend to reflect the severity of the patient's illness and the degree of AKI. Hyperkalaemia associated with ECG changes can be life-threatening and should be treated promptly with $10 \%$ calcium gluconate $(10 \mathrm{ml}$ slow intravenous push) to cardioprotect.
This should be followed by either an infusion of dextrose and insulin or nebulised salbutamol (10-20 mg) (but avoid if a history of cardiac arrhythmias) until a kaliuresis is achieved or renal replacement therapy (RRT) commenced. It is important to remember that appropriate modifications to drug dosing must be made depending on the degree of AKI.

Table 3(a). A recommended approach to clinical evaluation of a patient with acute kidney injury (AKI): patient history.

\begin{tabular}{|c|c|}
\hline \multirow[t]{2}{*}{ Previous history of kidney function } & Medical records/biochemistry lab \\
\hline & General practitioner \\
\hline \multirow[t]{6}{*}{ Comorbid conditions } & Chronic kidney disease \\
\hline & Cardiac disease \\
\hline & Vascular disease \\
\hline & Diabetes mellitus \\
\hline & Liver disease \\
\hline & Myeloma \\
\hline Family history & Kidney disease \\
\hline \multirow[t]{2}{*}{ Poor fluid intake } & Nausea/vomiting \\
\hline & Decreased functional capacity \\
\hline \multirow[t]{5}{*}{ Excessive fluid loss } & Fever \\
\hline & Overdiuresis \\
\hline & Diarrhoea/high stoma output \\
\hline & Haemorrhage \\
\hline & Burns \\
\hline \multirow[t]{3}{*}{ Systemic symptoms } & Rash (vasculitis) \\
\hline & Arthralgias (vasculitis) \\
\hline & Haemoptysis (vasculitis) \\
\hline \multirow[t]{2}{*}{ Urinary tract symptoms } & Prostate disease \\
\hline & Kidney calculi \\
\hline \multirow[t]{2}{*}{ Medications } & Nephrotoxic drugs \\
\hline & Radiocontrast \\
\hline
\end{tabular}

Table 3(b). A recommended approach to clinical evaluation of a patient with acute kidney injury (AKI): patient examination.

\begin{tabular}{ll}
\hline Skin & Rash \\
Volume status & Core temperature \\
& Heart rate \\
& Jugular venous pressure \\
& Blood pressure \\
& Peripheral perfusion \\
Cardiovascular status & Heart rate and rhythm \\
& Blood pressure \\
& Palpable bladder \\
Abdominal system & Increased abdominal pressure \\
Vascular system & Pulses/bruits \\
\end{tabular}

\section{Patients non-responsive to supportive therapy}

Patients whose AKI fails to respond to general supportive measures, or who may have been identified as potentially having AKI secondary to a cause other than hypoperfusion or ATN, should be discussed with the renal team. Specific therapy will depend upon the aetiology 
Table 4. Baseline investigations.

- U\&Es and bicarbonate

- $\quad$ LFTs ( $\uparrow$ bilirubin, $\uparrow A L T$ with liver failure, paracetamol overdose, leptospirosis)

- $\quad$ Bone profile ( $\uparrow$ calcium with myeloma)

- $\quad \mathrm{FBC}(\downarrow$ haemoglobin, $\downarrow$ platelets with HUS/TTP)

- Clotting screen

- CRP

- Urinalysis (blood \& protein may indicate ATN, GN or trauma from catheter)

- Urine microscopy (red-cell casts with GN, muddy brown granular casts with ATN) \pm urine culture

- $\quad$ Urine chemistry (see Table 6)

- $\quad$ KUB X-ray (if kidney calculi suspected)

- renal tract ultrasound (small kidneys with CKD, may be normal in the early phase of obstruction, consider repeating if high index of clinical suspicion)

$\mathrm{ALT}=$ alanine aminotransferase; ATN = acute tubular necrosis; CKD = chronic kidney disease; CRP = C-reactive protein; FBC = full blood count; GN = glomerulonephritis; HUS = haemolytic uraemic syndrome; KUB = kidneys, ureter and bladder; LFT = liver function test; TTP = thrombotic thrombocytopenic purpura; U\&E = urea and electrolytes.

\section{Table 5. Specialised investigations.}

- Immunology:

- ANCA (ANCA-associated vasculitis)

- Anti-GBM (Goodpasture's syndrome)

- ANA (lupus nephritis)

- complement (lupus nephritis, infectious endocarditis)

- myeloma screen

- Arterial blood gases ( $\uparrow$ lactate with liver failure, ischaemic bowel, metformin)

- Creatine kinase (rhabdomyolysis)

- $\quad$ LDH (HUS/TTP)

- Blood film (schistocytes with HUS/TTP)

- Toxicology screen (if overdose suspected)

- Renal biopsy (may be considered if there is suspicion of treatable glomerular or interstitial process)

ANA = antinuclear antibody; ANCA = antineutrophil cytoplasmic antibody; Anti-GBM = anti-glomerular basement membrane antibody; ATN = acute tubular necrosis; LDH = lactate dehydrogenase; HUS = haemolytic uraemic syndrome; TTP = thrombotic thrombocytopenic purpura.

Table 6. Urine chemistries used to differentiate pre-kidney acute kidney injury (AKI) and ischaemic intrinsic AKI (acute tubular necrosis (ATN)).*

\begin{tabular}{lcc} 
Urine chemistry & Pre-kidney AKI & ATN \\
\hline $\mathrm{FENa}^{+}(\%)$ & $<1$ & $>1$ \\
Urine $\mathrm{Na}^{+}(\mathrm{mmol} / \mathrm{l})$ & $<10$ & $>20$ \\
Urine osmolality $\left(\mathrm{mOsm} / \mathrm{kg} \mathrm{H}_{2} \mathrm{O}\right)$ & $>500$ & $<350$ \\
Urine/plasma urea & $>8$ & $<3$ \\
Urine/plasma creatinine & $>40$ & $<20$ \\
\hline
\end{tabular}

*Diuretics and dopamine may confound the interpretation of results.

$\mathrm{FENa}^{+}=$fractional excretion of sodium; $\mathrm{FENa}^{+}(\%)=$ (urine $\mathrm{Na} \times$ plasma creatinine)/(plasma $\mathrm{Na} \times$ urine creatinine) $\times 100$. $\mathrm{FENa}^{+}$is more accurate than urine $\mathrm{Na}^{+}$. of the AKI. Patients with an obstructed renal tract will require rapid relief of the obstruction: the longer the delay the poorer the outcome. Patients with biopsy proven vasculitis will require potent immunosuppressive therapy.

\section{Renal replacement therapy}

RRT provides a bridge while patients are receiving treatment for the underlying disease process. ${ }^{15,16}$ Absolute indications for commencing RRT include hyperkalaemia and pulmonary oedema resistant to medical therapy, uraemic pericarditis, encephalopathy and severe acidosis ( $\mathrm{pH}<7.1$ ). Initiation of RRT does not hasten recovery of AKI and is not without risk, but it is advisable to commence it prior to the development of overt metabolic and volume disturbance. There is no evidence to support the superiority of either intermittent or continuous RRT. A recent multicentre RCT has provided much-needed guidance on the dose of RRT required in critically ill patients with AKI. ${ }^{17}$

\section{Outcomes}

Despite significant advances in medical care, the overall mortality rate among

\section{Key Points}

New definitions and staging systems
are available for acute kidney injury
(AKI)

KEY WORDS: acute kidney injury, Acute Kidney Injury Network, biomarkers, ischaemia reperfusion injury, (RIFLE) staging system 
Table 7. Common complications associated with acute kidney injury.

\begin{tabular}{ll}
\hline Metabolic & Hyperkalaemia \\
& Metabolic acidosis \\
& Hypocalcaemia \\
& Hyperphosphataemia \\
Cardiovascular & Pulmonary oedema \\
& Arrhythmias \\
& Pericarditis \\
Gastrointestinal & Nausea \\
& Vomiting \\
& Gastritis \\
& Ulceration \\
& Malnutrition \\
Neurological & Seizures \\
& Mental status changes \\
Haematological & Anaemia \\
& Bleeding \\
Infectious & Pneumonia \\
& Septicaemia
\end{tabular}

patients with intrinsic AKI has remained at $50 \%$ for the past four decades. ${ }^{18}$ It has been argued that a failure to improve outcome may be explained by the use of more aggressive medical and surgical interventions in an ageing population. Most patients who survive following AKI will recover kidney function, ${ }^{19}$ but it is now recognised that a significant proportion of them will have subclinical functional defects and a smaller proportion will be at risk of developing CKD. ${ }^{20}$

\section{References}

1 Bellomo R, Ronco C, Kellum JA, Mehta RL, Palevsky P; Acute Dialysis Quality Initiative workgroup. Acute renal failure - definition, outcome measures, animal models, fluid therapy and information technology needs: the Second International Consensus Conference of the Acute Dialysis Quality Initiative (ADQI) Group. Crit Care 2004;8:R204-12.

2 Levin A, Kellum JA, Mehta RL; Acute Kidney Injury Network (AKIN). Acute kidney injury: toward an integrated understanding through development of a research agenda. Clin J Am Soc Nephrol 2008;3:862-3.

3 Chertow GM, Burdick E, Honour M, Bonventre JV, Bates DW. Acute kidney injury, mortality, length of stay, and costs in hospitalized patients. J Am Soc Nephrol 2005;16:3365-70.

4 Praught ML, Shlipak MG. Are small changes in serum creatinine an important risk factor? Curr Opin Nephrol Hypertens 2005; 14:265-70.

5 Hoste EA, Schurgers M. Epidemiology of acute kidney injury: how big is the problem? Crit Care Med 2008;36(4 Suppl):S146-51.

6 Uchino S, Bellomo R, Goldsmith D, Bates S, Ronco C. An assessment of the RIFLE criteria for acute renal failure in hospitalized patients. Crit Care Med 2006;34:1913-7.

7 Lameire N, Van Biesen W, Vanholder R. Acute kidney injury. Lancet 2008;372: 1863-5.

$8 \overline{\text { Brezis M }}$, Rosen S. Hypoxia of the renal medulla - its implications for disease. $\mathrm{N}$ Engl J Med 1995;332:647-55.

9 Macedo E, Bouchard J, Mehta RL. Renal recovery following acute kidney injury. Curr Opin Crit Care 2008;14:660-5.

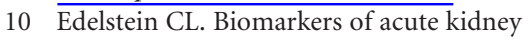
injury. Adv Chronic Kidney Dis 2008;15: 222-34.

11 Bonventre JV. Urine neutrophil gelatinaseassociated lipocalin as a marker of acute kidney injury in critically ill children. Nat Clin Pract Nephrol 2008;4:78-9.

12 Powell-Tuck J, Gosling P, Lobo DN et al. The British Consensus Guidelines on Intravenous
Fluid Therapy for Adult Surgical Patients (GIFTASUP). 2008 www.renal.org

13 McCullough PA. Radiocontrast-induced acute kidney injury. Nephron Physiol 2008;109:61-72.

14 Venkataraman R. Can we prevent acute kidney injury? Crit Care Med 2008;36(4 Suppl):S166-71.

15 Gibney N, Hoste E, Burdmann EA et al. Timing of initiation and discontinuation of renal replacement therapy in AKI: unanswered key questions. Clin J Am Soc Nephrol 2008;3:876-80.

16 Lewington AJ. Renal replacement therapy in the critically ill patient with acute kidney injury. Hemodial Int 2007;11:S39-43.

17 VA/NIH Acute Renal Failure Trial Network, Palevsky PM, Zhang JH, O'Connor TZ et al. Intensity of renal support in critically ill patients with acute kidney injury. $\mathrm{N} \mathrm{Engl} \mathrm{J}$ Med 2008;359:7-20.

18 Hoste EA, Kellum JA. Incidence, classification, and outcomes of acute kidney injury. Review. Contrib Nephrol 2007;156:32-8.

19 Goldberg R, Dennen P. Long-term outcomes of acute kidney injury. Adv Chronic Kidney Dis 2008;15:297-307.

20 Schiffl H, Fischer R. Five-year outcomes of severe acute kidney injury requiring renal replacement therapy. Nephrol Dial Transplant 2008;23:2235-41.

Address for correspondence:

Dr A Lewington, Renal Department,

St James's University Hospital, Beckett Street, Leeds LS9 7TF. Email: Andrew.Lewington@leedsth. nhs.uk 